\title{
Las políticas de reparación simbólica a las víctimas del conflicto armado interno: Avances en el caso de Accomarca, Ayacucho
}

Sección ESTUDIOS

RECIBIDO: 25/08/2021

APROBADO: 15/09/2020

PUBLICADO ONLINE: 30/12/2021

\author{
Geraldine Yolanda Garrido Espíritu \\ Universidad Nacional Mayor de San Marcos \\ geraldine.garrido@unmsm.edu.pe \\ https://orcid.org/0000-0002-1651-6963
}

\section{RESUMEN}

En el 2003 se realizó la entrega del Informe Final de la Comisión de la Verdad y Reconciliación que recogió alrededor de 7,300 testimonios de víctimas del conflicto armado interno vivido entre los años 1980 y 2000 en el Perú. A partir de lo recogido, se brindó recomendaciones y reflexiones con la idea de generar una nueva ciudadanía y reformar las instituciones para prevenir la violencia y respetar los derechos humanos. Sin embargo, estas acciones necesitan un proceso de reconocer a las víctimas y brindarles un debido proceso de justicia y reparación.

En el presente artículo, se presenta parte de la investigación que sigue en proceso sobre el programa de reparaciones simbólicas en el distrito de Accomarca de la región de Ayacucho, el cual sigue a la espera de la construcción de un lugar de memoria para recordar, albergar y encontrar las memorias, vivencias y luchas de su comunidad.

PALABRAS CLAVE: Conflicto armado interno, políticas de memoria y reparación, lugares de memoria, reparación simbólica, Perú, Argentina, Chile, Accomarca.

\section{Policies of symbolic reparation to the victims of the internal armed conflict: Progress in the case of Accomarca, Ayacucho}

\section{ABSTRACT}

In 2003, the Final Report of the Truth and Reconciliation Commission was delivered, which gathered around 7,300 testimonies from victims of the internal armed conflict that took place between 1980 and 2000 in Peru. Based on what was collected, recommendations and reflections were provided with the idea of generating a new citizenship and reforming institutions to prevent violence and respect

(c) Los autores. Este artículo es publicado por la Revista de Sociología de la Facultad de Ciencias Sociales, Universidad Nacional Mayor de San Marcos. Este es un artículo de acceso abierto, distribuido bajo los términos de la licencia Creative Commons Atribución 4.0 Internacional (CC BY 4.0) [https://creativecommons.org/licenses/by/4.0/deed.es] que permite el uso, distribución y reproducción en cualquier medio, siempre que la obra original sea debidamente citada de su fuente original. 
human rights. However, these actions need a process of recognizing the victims and providing them with a suitable process of justice and reparation.

This article addresses part of the investigation that is still in process on the symbolic reparations program in the Accomarca district of the Ayacucho region is presented, which continues to await the construction of a place of memory to remember, house and find the memories, experiences and struggles of their community.

KEYWORDS: Internal armed conflict, policies of memory and reparation, places of memory, symbolic reparation, Perú, Argentina, Chile, Accomarca.

\section{Introducción}

E 28 de agosto del 2003 se realizó la entrega del Informe Final de la Comisión de la Verdad y Reconciliación en el 2003 al ex mandatario Alejandro Toledo. Durante la ceremonia, Salomón Lerner, presidente de la comisión, señaló que más de 69 mil peruanos y peruanas fueron víctimas del conflicto armado interno. Asimismo, reveló que como país debemos aprender a vivir con estas cifras pues es momento de asumir nuestra responsabilidad como sociedad y Estado.

La investigación dio como resultado nueve tomos que narraban los hechos de horror vividos por ciudadanas y ciudadanos de diferentes partes del país, sin embargo, la gran cantidad de testimonios recogidos en zona rurales y de difícil acceso eran escalofriantes. De un momento a otro, las víctimas y sus familiares empezaron a reunirse y relatar, las organizaciones se pusieron de pie y brindaron testimonios que habían recogido por su cuenta. Y cuando no fue suficiente esto, se empezaron a realizar en señal abierta las audiencias públicas transmitidas por el canal del Estado. A medida que pasaba el tiempo, una pregunta retumbaba en los comisionados y la población: ¿Dónde había estado el Estado para todas estas víctimas?

A partir de lo mencionado, los gobiernos sucesivos empezaron a tomar acciones para reestructurar las relaciones entre las instituciones públicas y ciudadanía que fueron afectadas por la violencia y desigualdad. La implementación y ejecución de políticas de reparación a las víctimas comenzaron a ser un punto en la agenda nacional, sin embargo, tras más de quince años de la entrega de la Comisión de la Verdad, las luchas por la verdad persisten. Por ello, el presente artículo considera pertinente y necesario abordar de qué forma se ha llevado a cabo las políticas de reparación en el Perú, tras la búsqueda de justicia por más de 40 años de las víctimas y sus familiares.

El presente artículo tiene como objetivo presentar parte de una investigación personal sobre el programa de reparaciones simbólicas en el distrito de Accomarca de la región de Ayacucho. Comunidad que sigue a la espera de la construcción 
de un lugar de memoria y busca sumar espacios de diálogo y encuentro. Pues no olvidemos que en nuestro país tenemos memorias contra memorias: la lucha ciudadana defendiendo los derechos humanos y el debido proceso mientras que, por otro extremo, la memoria mezquina y hegemónica que no reconoce los actos de violencia.

\section{¿Por qué reparar?}

Cuando se habla del conflicto armado en el Perú, suele entenderse como un periodo de tiempo pasado y ajeno. En algunos casos, es preferible no mencionarlo o removerlo porque no es solo doloroso y confuso, sino que depende la posición en que nos encontremos para entender sus complejidades, como sostiene Reátegui (2010). Sin embargo, lo cierto es que muchas veces, socialmente hablando, ha sido más cómodo no nombrarlo y voltear la página.

Pero qué pasa cuando aún se busca a alguien, cuando no se ha podido enterrar a una madre, padre, hermano o hermano, es más, no se tiene la certeza de quiénes se lo llevaron y sí se tiene, estas personas están libres y siguiendo con su vida. Entonces ¿cómo se puede reparar la desaparición o muerte de una persona? ¿Es realmente posible hacerlo? ¿Se puede hacer justicia en estos casos? Estas preguntas las dejaré abiertas por el momento.

A raíz de los hechos de violencia producidos en los años 1980-2000 que generaron graves consecuencias en la dignidad e integridad de las personas que fueron torturadas, desplazadas forzadamente y encarceladas injustamente mencionadas en la Comisión de la Verdad y Reconciliación, que en adelante será CVR, se puede desglosar que es importante también señalar que muchos de estos casos tuvieron mayor incidencia en los sectores más pobres del Perú, ya que muchas veces no han tenido una presencia total por parte del Estado y a causa de eso han sufrido la indiferencia y muchas veces la soledad para poder afrontar la violencia (CVR, 2008, p. 17).

El tema de la reparación es bastante complejo debido a que no se puede compensar la pérdida o la desaparición de una persona, sin embargo, desde la Comisión de la Verdad y Reconciliación, "la reparación es un gesto que reconoce y reafirma la dignidad de las personas" (CVR, t. IX, 2003, p. 141). En la historia del Perú, la violencia no es un tema nuevo, sin embargo, tras lo investigado por la Comisión de la Verdad se logró hablar de un pasado -reciente- el cual aún tenía muchos temas por cerrar, pero sobre todo heridas de invisibilización que debían ser reconocidas, escuchadas y reparadas (CVR, t. IX 2003, p. 316). Asimismo, el trabajo 
de las instituciones de derechos humanos hizo del tema de las reparaciones una de las principales demandas, logrando así que en diciembre del 2006 se promulgase la Ley N 28592 Plan Integral de Reparaciones (PIR) (Congreso de la República, 2006).

Con ella, se crea el Consejo de Reparaciones, que en adelante se llamará CR, cuya institución sería la encargada de elaborar el Registro Único de Víctimas (RUV). Este registro permitiría tener una relación oficial de las víctimas del conflicto armado a nivel nacional. Y, por otro lado, podrían acceder a los programas de reparación que se estaban empezando a implementar con la normativa (Congreso de la República, 2006). Sin embargo, estas acciones no son exclusivas de nuestro contexto nacional, a continuación, se presenta de forma breve algunas políticas y acciones de dos países hermanos: Argentina y Chile.

Bajo el Decreto 187 en el gobierno de Raúl Alfonsín en Argentina, el 15 de diciembre de 1983 se dio inicio a la Comisión Nacional sobre las Desaparición de Personas (CONADEP). Su mandato estuvo ceñido a investigar las violaciones contra los derechos humanos ocurridos entre 1976 y 1983. Además, tenía la aprobación para recibir las denuncias sobre desapariciones y secuestros para elaborar un documento de trabajo.

La investigación pudo recoger testimonios que narraron la historia de detenidos y desaparecidos durante nueve meses en la capital, así como filiales. La labor fue muy difícil, sin embargo, el ímpetu para esto era motivada para aportar al surgimiento de una sociedad democrática que no volviera a vivir estos años oscuros. Ernesto Sábato presidio la CONADEP que estaba integrada por doce comisionados. La representación incluía a iglesias (católica, judía y metodista) y tres diputados que llegaron a pedido del presidente de la época. Además, la integraron alrededor de sesenta investigadores.

Al igual que la Comisión peruana, en el caso argentino la identificación de responsables no era la misión encomendada de la investigación, sin embargo, mencionó las acciones y vulneración de derechos de organismos como el Estado y grupos violentos. Se contó con la ayuda de organismos de DDHH para la recepción de testimonios.

La comisión entregó finalmente el nombre de 1351 opresores el 20 de septiembre de 1984 con más de 50 mil carillas y el 28 de noviembre, un mes después, se presentó el libro "Nunca más". Los resultados fueron atroces: más de ocho mil personas desaparecidas - detenidas, así como más de 300 centros de detención. (Espinoza, Ortiz y Rojas, 2003, p. 14 -16).

Tras esto, se establecen recomendaciones y dirigidas a dos ámbitos. La primera promover iniciativas para prevenir y reparar la repetición de la violencia contra los 
Derechos Humanos. La segunda, recomendaciones enfocadas en la justicia para generar búsquedas exhaustivas y procesos judiciales contra las y los responsables.

Como parte de sus políticas de reparación, se implementaron normas y leyes dirigidas a los hijos y/o familiares de los detenidos y desparecidos, esto incluía la asistencia económica, becas de estudio, asistencia social, puestos de trabajo y se tomen medidas de atención por consecuencias psicológicas por la pérdida de familiares. Hasta el año 2011 habían contabilizado un total de 50 políticas públicas en el ámbito de la búsqueda de la verdad, memoria y reparación representada en reestructuración de instituciones y creación de otras, espacios de memoria y encuentro, así como modificación en el ámbito educativo para el dialogo de estos temas (Centro de Derechos Humanos, 2011, p. 27).

Por otro lado, en el caso chileno el 25 de abril de 1990 se decreta la conformación de la Comisión Nacional de Verdad y Reconciliación (CNVR) bajo el mandato de Patricio Aylwin. Sus funciones fueron determinadas en el Decreto Supremo N³55 que establecía generar un cuadro completo de las violaciones contra los Derechos Humanos, buscar antecedentes que resuelvan los paraderos de las y los desaparecidos y, recomendar medidas de reparación y reivindicación de justicia para lograr una reconciliación en la sociedad chilena. Sin embargo, tenía prohibido pronunciarse sobre responsabilidades individuales pues esto solo les competía a los tribunales, por ello, lo investigado le fue enviado a los magistrados para las correspondientes acciones.

Como parte de los casos que encontró, sostiene Espinoza, Ortiz y Rojas, (2003) se puede mencionar la desaparición de personas, torturas y ejecuciones por parte del Estado. El periodo que abarcó de investigación fue de siete años (1973-1990) la cual demoró catorce meses entre la indagación y escrito del informe. Finalmente, fue entregado en 1991 dividido en tres tomos consignando 3400 casos y en 1992, mediante la Ley N 19123 se creó la Corporación Nacional de Reparación y Reconciliación (CRR) para implementar las recomendaciones de la Comisión que consideraban lo siguiente: reivindicación y reparación simbólica, bienestar social y de carácter legal a las instituciones de justicia Las políticas de reparación en Chile siguieron parámetros similares de Argentina sobre la no repetición de actos de violencia y el respeto a los derechos humanos. Además, se hizo hincapié en que el poder judicial y otras instituciones estatales cumplan con su rol garante de los derechos en materia constitucional, penal y procesal penal. Esto genera también que se mantenga la colaboración en la búsqueda de las víctimas y eliminar por completo el ocultamiento de información. Según el Centro de Derechos Humanos de Chile 
(2011), se cuenta con más de 30 políticas sobre memoria y reparación que incluyen espacios de memoria, instituciones especializadas y reconocimientos públicos.

\section{Los programas de reparación en el Perú}

Siguiendo lo mencionado, Jelin (2002) señala que, durante periodos que suceden en épocas de violencia y traumas donde se pusieron en suspenso los derechos humanos, emerge un nuevo clima de búsqueda de justicia y verdad donde diversas memorias y sus interpretaciones son elementos fundamentales para la construcción de identidades individuales y colectivas. Por ello:

[...] las memorias de quienes fueron oprimidos y marginalizados [...] surgen con una doble pretensión, la de dar la versión "verdadera" de la historia a partir de su memoria, y la de reclamar justicia. En esos momentos, memoria, verdad y justicia parecen confundirse y fusionarse, porque el sentido del pasado sobre el que se está luchando es, en realidad, parte de la demanda de justicia en el presente". (Jelin, 2005, p. 23)

En las últimas cuatro décadas, el conjunto de medidas para la restitución de derechos fundamentales se ha conceptualizado como justicia transicional "definida como aquella concepción de la justicia asociada a períodos de cambio político" (Teitel, 2003, p. 69). A partir de lo mencionado, se observa un trabajo articulado con la sociedad, en este caso, actores sociales y organismos demandantes de derechos humanos quienes han tenido un rol activo y constante en los procesos de memoria y justicia llevando a instancias internacionales las demandas de las víctimas. En base a las ideas planteadas por Velásquez, González (2003) se puede decir que la relación entre Estado y sociedad mediante la participación ciudadana está sujeta a la esfera social y la demanda. La palabra reparación según el Diccionario de la Real Academia Española (2021) proviene del latín reparare que significa "arreglar o recomponer algo que se ha roto".

Espinoza, Ortiz y Rojas (2003) sostienen que las reparaciones se han pensado en países donde ocurrió hechos que vulneran los Derechos Humanos. Se debe mencionar que cada país tiene una responsabilidad ética y política puesto que existen normas y principios internacionales que respaldan las reparaciones a las personas víctimas de violencia durante conflictos o tiempos de guerra.

Adicionalmente, muchos organismos o colectivos de derechos humanos señalan que el concepto de reparación tiene "una combinación de verdad, justicia y 
apoyo a los sobrevivientes" (p. 87). De forma sucinta, la reparación está sujeta a las categorías de: verdad, justicia, reconciliación, prevención y promoción. En el Perú, como en otros contextos donde se vivieron periodos de violencia, el auge de la memoria se relaciona con la lucha política que se desarrolla en el plano de la cultura entre formas contrapuestas de entender y valorar el horror de los años de violencia (Barrantes y Peña, 2010). Existe justamente, los conflictos entre "las memorias" se dividen entre quienes fueron víctimas y quieren sustentan la memoria salvadora que justifica "el costo inevitable que se tuvo que pagar para alcanzar derrotar el terrorismo" (Degregori, Portugal y Salazar, 2015, p. 15-16).

Como se mencionó, con el Decreto Supremo N 011-2004-PCM se crea la Comisión Multisectorial de Alto Nivel (CMAN), quien estaba encargada del "Seguimiento de las Acciones y Políticas del Estado en los ámbitos de la Paz, Reparación Colectiva y la Reconciliación Nación"1.

El Marco Normativo del Plan Integral de Reparaciones - PIR para las víctimas de la violencia ocurrida durante el período de mayo de 1980 a noviembre de 2000, conforme a las conclusiones y recomendaciones del Informe de la Comisión de la Verdad y Reconciliación. (Congreso de la República, 2006, p. 2)

De esa forma, la CMAN se convierte en el agente encargado de la implementación y ejecución de los 7 programas de reparación que está en el Artículo 2.- Componentes del Plan Integral de Reparaciones (Congreso de la República, 2006, pág. 2): Programa restitución de derechos ciudadanos, reparaciones en educación, salud, colectivas, simbólicas, programa de promoción y facilitación al acceso habitacional, entre otros.

Por otro lado, desde el año 2012, ambas instituciones, la CMAN y el RUV dependen del Ministerio de Justicia y Derechos Humanos. Finalmente, las personas que pueden acceder al plan de reparaciones deben cumplir ciertos requisitos:

Para efecto de la presente Ley son consideradas víctimas las personas o grupos de personas que hayan sufrido actos u omisiones que violan normas de los Derechos Humanos, tales como desaparición forzada, secuestro, ejecución extrajudicial, asesinato, desplazamiento forzoso, detención arbitraria, reclutamiento forzado, tortura, violación sexual o muerte, así como a los familiares de las personas muertas y desaparecidas durante el período comprendido en el artículo 1 de la presente Ley. (Congreso de la República, 2006, p. 2)

1 Según la finalidad que tiene la labor de la Comisión Multisectorial de Alto Nivel (CMAN) expuesta en su pág. Web: https://cman.minjus.gob.pe/quienes-somos/ . (última consulta: 14/11/2021) 
Ahora, los programas de reparación involucran otras instancias. El Programa de Restitución de Derechos ciudadanos involucra la regularización jurídica de personas requisitoriadas indebidamente, anulación de antecedentes policiales, judiciales y penales para indultados inocentes, regularización de documentos (DNI) y exoneración de tasas judiciales administrativas registrales, municipales. El programa de Reparación en Educación exonera de pagos administrativos, implementa becas y brinda acceso y restitución a la educación básica regular. La reparación en salud permite la atención de víctimas e integración al SIS. Por otro lado, las reparaciones colectivas abordan la recuperación y reconstrucción infraestructura económica, productiva y comercio, así como el desarrollo de capacidades humanas y acceso a oportunidades económicas. Las reparaciones en promoción y facilitación al acceso habitacional permiten la construcción de viviendas, apoyo para el saneamiento legal de la propiedad de la vivienda y apoyo a las familias desplazadas.

Finalmente, llegamos a las reparaciones simbólicas: estas siguen los lineamientos de gestos públicos por los representantes de los Poderes del Estado, actos de reconocimiento a víctimas o que conduzcan a la reconciliación y recordatorios. Según el artículo $32^{\circ}$ del Reglamento del Plan Integral de Reparaciones, las modalidades del Programa de Reparaciones Simbólicas son "los gestos públicos, actos de reconocimiento y actos que conduzcan hacia la reconciliación". (CMAN, 2013, p. 6).

En el caso de los gestos públicos, estos están referidos a las disculpas públicas por parte de los representantes del Estado dirigida a las familias, comunidades y poblaciones afectadas. Puede ser por medio de una carta, evento, reunión, mesa de diálogo o ceremonias públicas. Sin embargo, es importante señalar que para los familiares es muy importante el tema de las disculpas es el primer paso para reconocer la violencia de los años ochenta.

Posterior a los gestos, se llevan a cabo los actos de reconocimiento, que implica el encuentro entre quienes vivieron este periodo de violencia como los líderes, autoridades y civiles; así como miembros de las Fuerzas Armadas, Policía Nacional donde se reafirma el compromiso de la defensa de los derechos de las comunidades.

El Registro Único de Victimas (RUV) permite también hacer un seguimiento de los casos y las familias, es más, muchos de quienes están inscritos han solicitado que la lucha de sus familiares sea reconocida en sus comunidades. Por ejemplo, los recordatorios como fechas, lugares públicos son también parte de este proceso de reparación simbólica.

Estos recordatorios hacen referencia a la revalorización de lugares de memoria ubicados en la comunidad, los cuales requieren gestión y coordinación con los gobiernos locales y regionales que, a través de acciones para la implementación, 
ejecución, mantenimiento, mejora y difusión llegue a cumplir con las necesidades de la población.

De esta forma, "cada espacio de memoria pasa por un proceso de diálogo y acuerdo, y tiene diversos sentidos para quienes están involucrados en su creación; de igual forma, cabe resaltar que la población tiene espacios y modos de rememorar propios" (CMAN, 2013, p. 11). Cabe añadir que, las modalidades de la reparación simbólica están acompañadas del trabajo en conjunto con otras instancias del Estado.

Las reparaciones simbólicas tienen muchas formas de ejecutarse pues no existe una guía formal o pasos a seguir, debido a esto, su forma de implementarse permite que las víctimas y familias puedan ejercer la libertad de solicitar lo que vean conveniente para cada una.

\section{5: El inicio de la lucha de Accomarca}

El distrito de Accomarca pertenece a uno de los ocho distritos que conforma la provincia de Vilcashuamán, en la región Ayacucho. Fue fundado el 29 de enero de 1965 y tiene una población de 886 personas (INEI, 2017).

El 14 de agosto de 1985, la patrulla Lince dirigida por el subteniente del ejército Telmo Ricardo Hurtado asesinó a 62 comuneros quienes pertenecían al Distrito Accomarca en la provincia de Vilcashuamán, Ayacucho. Este hecho de violencia y vulneración de Derechos Humanos fue parte de las acciones del gobierno de Alan García para frenar el terrorismo que azotaba la sierra del país. Este plan antiterrorista fue organizado y planificado desde la Sub Zona de Seguridad Nacional Número 5 y se le llamó el Plan Operativo Huancayoc. (CVR, t. VII, 2003, p.155)

En el transcurso de los años '80, Sendero Luminoso había centrado sus bases senderistas en las provincias de Cangallo y Víctor Fajardo: debido a esto, fueron consideradas como sus zonas de "comité central" o espacio donde se encontraba parte de la cúpula de sendero y se planificaban las acciones (CVR, t. VII, 2003). Por ello, impusieron la formación de "comités populares" y la revocación de autoridades mediante la violencia (CVR, t. VII, 2003). Sin embargo, no todas las autoridades dejarían sus cargos, y, por el contrario, intentaban fortalecer la organización social mediante el trabajo de las rondas campesinas y posteriormente los comités de autodefensa (CVR, t. II, 2003). Uno de estas organizaciones surgió en Accomarca.

En un primer momento algunas autoridades locales fueron aceptadas por sendero ya que al permitirlas se consideraban seguras para el ejército y alejaba el 
establecimiento de las bases militares, sin embargo, bastaba que alguna autoridad no quisiera renunciar a su cargo para que fuera asesinado.

Sin embargo, una de las características centrales de los primeros años del conflicto, es que no ocurrió un cambio inmediato en la vida cotidiana de la comunidad, sino que ésta fue afectándose de manera gradual. Así, durante los primeros años, en Accomarca las actividades económicas y educativas siguieron su curso normal, a pesar de lo cual, algunas personas de la comunidad empezaron a mostrarse en desacuerdo con el PCP-SL porque se les prohibía cuestionar a los dirigentes, se impuso un sistema de producción distinto al normal y eran obligados a asistir a las asambleas. (CVR, t. VII, 2003, p. 155)

De esta forma, el año 1982 transcurrió hasta que, en los años siguientes, las acciones de sendero fueron cada vez más violentas donde la negación por parte de la ciudadanía era castigada con la muerte, precisando estos actos de violencia, se recoge lo siguiente del mismo tomo mencionado líneas arriba:

Ante esta situación, en 1983 se iniciaron las primeras incursiones por parte del Ejército en las denominadas "zonas liberadas", o lugares donde Sendero pretendía haber instalado "comités populares". Las incursiones a los poblados tenían como resultado la detención y muerte a discreción de campesinos. Sendero Luminoso se había convertido en un enemigo invisible que las fuerzas del orden no podían identificar, lo que resultaba en acciones arbitrarias contra el conjunto de los habitantes, que eran culpabilizados por asociación. (p. 156)

Sumado a todo esto, el ejército comenzó a realizar sus operativos de forma más rigurosa, y lamentablemente, bastaban las sospechas para condenar a un poblador o comunidad entera.

Las autoridades del Ejército del departamento de Ayacucho estaban seguras que sectores de la población del distrito de Accomarca estaban vinculados al PCP-SL, que existía una "Compañía Accomarca" de Sendero Luminoso, y que en la parte baja de este distrito - Quebrada de 156 Huancayoc - funcionaba una"escuela popular". El Ejército arribó a dichas conclusiones luego de que, en la primera semana del mes de agosto de 1985, se produjeran enfrentamientos con columnas de Sendero en las alturas de Sarhua, Caracha y Putuccasa del distrito de Huancasancos, que habían causado 25 bajas entre los senderistas. En estos enfrentamientos fueron detenidos un número de presuntos subversivos que habrían aceptado pertenecer a la "compañía Accomarca", la cual, según informaron, estaba conformada por un total de 32 hombres organizados en cinco pelotones. (p. 156-157) 
Tras este hecho, se decretó ejecutar Plan Operativo Huancayoc

A las 6:30 de la mañana aproximadamente del 14 de agosto, el Sub Teniente Telmo Hurtado y los integrantes de su patrulla llegaron a la Quebrada de Huancayoc y Lloqllapampa [...]. La patrulla ingresó por varios frentes realizando disparos de arma de fuego con el supuesto fin de evitar la fuga de los pobladores [...]. A pesar de que el Ejército realizó una búsqueda exhaustiva de material subversivo, [...] no se encontraron armas, municiones, explosivos o propaganda de Sendero Luminoso. Luego de la búsqueda, todos los pobladores fueron reunidos en la zona conocida como Hatunpampa [...] 159 pobladores se encontraban [...] el propio Telmo Hurtado, además de dar la orden de disparar, lanzó una granada provocando una explosión y el incendio de los lugares donde se encontraban las personas detenidas. (CVR, t. VII, 2003, p. 159-160)

A partir de 1985, se abrió un proceso de investigación contra Hurtado por la justicia militar que duró cuatro años, pero fue absuelto por el delito de homicidio calificado y sólo se le condenó por abuso de autoridad ya que se consideró que la masacre había ocurrido en el contexto de combatir el terrorismo. La pena fue de 6 años de privación a su libertad. Sin embargo, en 1995, Hurtado y otros implicados se vieron beneficiados por la Ley de Amnistía promulgada por el congreso durante el gobierno de Alberto Fujimori. En el año 2002 la Corte Interamericana de Derechos Humanos falla a favor del caso Barrios Altos y se anula la mencionada ley, dando pie a que se realice un nuevo juicio en el caso de Accomarca. Tras esto, Hurtado huye durante 5 años a Miami hasta que es detenido por las autoridades y en el año 2011 se realiza su extradición siendo conducido al penal Castro Castro. (Huber \& Del Pino, 2015).

Sin embargo, Accomarca a pesar de ser uno de los casos más emblemáticos del país, sigue a la espera de un lugar de memoria como parte de las reparaciones simbólicas solicitada por la comunidad. Cabe añadir que en el año 2017, la ex ministra de Justicia y Derechos Humanos, María Soledad Pérez Tello, inauguró el Santuario Ecológico de Lloccllapampa, en la provincia de Vilcashuamán, Ayacucho. Además de brindarse una campaña de salud y atención legal gratuita a cargo de brigadas de Defensa Pública del sector, que orientaron a los pobladores sobre temas de familia, conciliación y de víctimas de abusos. Sin embargo, esto no es suficiente.

Desde la Asociación Hijos de Accomarca ubicada actualmente en el distrito de Ate Vitarte en la región de Lima se viene armando un boceto para la construcción del Lugar de Memoria en Accomarca, el cual, está pensado estar ubicado en el mismo terreno donde funcionó un cuartel militar de la zona. Esto con la finalidad de poder velar a sus desaparecidos en el lugar donde fueron llevados y vistos por última vez. 
Este proyecto, desde antes de la pandemia quedó paralizado pues los temas administrativos y la falta de apoyo por parte del municipio y representantes estatales generó malestar en la comunidad que lejos de olvidarlo, sigue manteniendo la unión de las familias por lograr una visibilización y reconocimiento.

\section{A modo de conclusión}

En la historia de cada país, región o localidad han existido diferentes hechos históricos: algunos buenos que aportaron al crecimiento de mejores condiciones de vida para sus ciudadanos, y otros, nefastos y crueles que han dejado huellas que hasta el día de hoy generan dolor e impotencia.

Según Rodríguez (2013), cada Estado tiene la obligación de investigar y sancionar, según sus leyes y normas, a los responsables de violentar Derechos Humanos. Además, para poder esclarecer estos terribles hechos se deben conformar espacios donde "el poder político aliente o apoye activamente una investigación seria de abusos cometidos en el pasado" (p. 268).

De esta forma, las Comisiones de Verdad se fueron implementando como mecanismos no judiciales, pero sí de carácter oficial que constatan hechos de violencia donde han ocurrido violaciones de Derechos Humanos a lo largo de ciertos períodos de años como se señala:

A pesar de la necesaria existencia de estos mecanismos, orientados todos ellos a procesar a los responsables de delitos contra los derechos humanos en el extranjero (...) lo deseable sería que estos procesos fueran celebrados en el país que sufrió los crímenes que van a juzgarse, no ya porque habría un mejor acceso a pruebas, testimonios de los supervivientes e imputados, sino porque otorgaría al país un fuerte aliciente para iniciar una justa y fuerte transición basada en los principios de justicia, verdad y reparación, así como confianza en sus tribunales nacionales. (Rodríguez, 2013, p. 268)

La investigación de Espinoza, Ortiz y Rojas (2003) señalan que la primera comisión surgió en Argentina, en el año 1983 debido a los crímenes que se estaban cometiendo durante la dictadura militar. Siete años después, Chile comenzaría este proceso también. Concluyen que "uno de estos deberes es el de investigar y sancionar a los responsables de tales hechos" (pp. 229-230). También Rodríguez (2013) refiere que es esencial que una Comisión de Verdad tenga la libertad de investigar el período de tiempo donde se dieron la mayor cantidad de violaciones 
contra los Derechos Humanos sin dejar de lado temas de discriminación, racismo y otros temas correspondientes.

Sin duda, la creación de comisiones de verdad marcó un precedente histórico como señala Huber y Del Pino (2015) porque su conformación por múltiples personalidades de diversas identidades políticas e ideológicas parecía coincidir en la búsqueda de la reconstrucción democrática y la verdad histórica, anteponiendo los derechos humanos y la justicia para la reparación, como la base de este nuevo Estado.

Como lo mencionan tanto Degregori, Portugal, Salazar (2015) como Burbano y García (2016) las reparaciones involucran una serie de criterios y requisitos los cuales deben adecuarse a las necesidades de la ciudadanía. Entre ellos, podemos rescatar los siguientes: alcance, cobertura, amplitud, complejidad coherencia interna y externa, y finalidad.

De esta forma, los programas de reparación pueden ejecutarse de diferentes formas. Como mencionó el Centro de Derechos Humanos en Santiago de Chile, en el caso de Argentina se crearon alrededor de 50 políticas y en la experiencia chilena se han contabilizado 31.

La reparación no es un proceso netamente institucional, sino colectivo. La participación de organizaciones acompaña a los organismos estatales, quienes, como se ha visto líneas arriba, deben cumplir un mandato de atención. Sin embargo, el estudio de Sinthya Rubio (2013) ha recogido las críticas al Plan Integral de Reparaciones (PIR) implementado en el Perú desde el año 2004.

[...] no se tuvieron en cuenta las experiencias locales y los planes regionales que ya se encontraban en marcha; que no se dio un enfoque de género e interculturalidad de manera adecuada, [...]. Igualmente, no se incluyó a los pueblos indígenas que fueron muy afectados por el conflicto armado interno, además está pendiente la reparación integral a las personas en situación de desplazamiento por la violencia, ellos también deben ser incorporados en las reparaciones colectivas. (p. 60)

Entre sus aportes, señala que es importante relacionar el contexto social, político y económico de las comunidades y poblaciones agredidas. No se puede crear 0 implementar en medidas de atención sin antes conocer la realidad y necesidades. Menos si parte de estas poblaciones han luchado por conquistar espacios de organizaciones sociales y hasta conseguir disputas legales internacionalmente.

De esta forma, el programa de reparaciones a pesar de haberse instalado como una política pública de memoria durante casi 20 años, todavía presenta desafíos en su implementación y ejecución. 
Sin embargo, es necesario conocer el desarrollo de los mismos. Podemos concluir que las reparaciones no solo son compromisos sino necesidades y exigencias ciudadanas. Por ejemplo, las reparaciones simbólicas mediante los espacios o lugares de memoria se han dado en forma de conmemoración ya que fueron escenario de violencia. Algunos han sido institucionalizados por la misma ciudadanía como el Mural 15 años de impunidad de la desaparición de 9 campesinos de Santa y La Hoyada (Reátegui, 2010) y seguro, otros lugares que se encuentran en la clandestinidad. Entonces, las reparaciones pueden desarrollarse civilmente, pero esto no significa que no deba ser un punto de agenda para el Estado.

En un artículo de Bunselmeyer (2016) fueron entrevistadas 160 personas, a quienes se les consultó sobre su percepción respecto a la ejecución de las reparaciones en sus localidades. La mayoría de la gente entrevistada no está contenta con el programa de reparaciones. En todo caso, están a favor de la idea de reparaciones, pero no cómo se ha llevado y se lleva a cabo la implementación "porque las expectativas no son cumplidas, las víctimas y sus familiares se sienten otra vez desilusionados del Estado.

Después de todo lo explicado, se considera clave seguir investigando la ejecución de las políticas de reparación y abordarlo visibiliza que aún persisten las memorias en conflicto: la lucha de quienes defienden los derechos humanos y procesos de reparación, por otro extremo, la memoria mezquina y hegemónica que tarda tanto en responder que ya no se siente como justicia. Sin embargo, las propias víctimas de la violencia han sabido organizarse y tener cierta relevancia política con sus discursos de justicia ("ni olvido, ni perdón"), reparaciones y reconciliación. Ante esto, adentrarse en investigaciones sobre la violencia y el conflicto armado interno responde también a un compromiso ciudadano. 


\section{Bibliografía}

Barrantes, R., y Peña, J. (2010). Los sitios de memoria. Procesos sociales de la conmemoración en el Perú. Lima: IDEHPUCP.

Bunselmeyer, E. (2016). ¿Cómo reparar? El impacto de las reparaciones y el tejido social en once comunidades andinas. Revista Memoria, (24), 22-29.

Burbano, N., y García, R. (2016). La reparación de víctimas de conflictos armados: experiencias internacionales. Inciso 18 (2), 57-67.

Centro de Derechos Humanos. (2011). Políticas Públicas de Verdad y Memoria en 7 países de América Latina. Santiago de Chile: Centro de Derechos Humanos.

Comisión de la Verdad y Reconciliación (CVR). (2003). Informe Final de la Comisión de la Verdad y Reconciliación, t. VII, IX. Lima: CVR.

Comisión de Entrega de la CVR (2008). Hatun Willakuy. Versión abreviada del Informe Final de la Comisión de la Verdad y Reconciliación: Perú. Lima: CVR.

Comisión Multisectorial de Alto Nivel. (junio de 2013). Plan Integral de Reparaciones. Lineamientos Generales del Programa de Reparaciones Simbólicas. Lima, Lima, Perú: Congreso de la República.

Congreso de la República. (28 de setiembre de 2006). Ley N²8592. Ley que crea el Plan Integral de Reparaciones - PIR. Lima, Perú: Congreso de la República.

Crenzel, E., y Vinyes, R. (2009). Espacios, memoria y territorio: un memorial en red en Cataluña. En R. Vinyes, El Estado y la memoria. Gobiernos y ciudadanos frente a los traumas de la historia. Barcelona: RBA.

Cuzcano, F., Solis, M., y De los Rios, C. (2017). Constructores de Pazen Ayacucho. Sistematización de la experiencia. Huamanga: Centro Loyola Ayacucho.

Degregori, C., Portugal, T., Salazar, G., y Aroni, R. (2015). No hay mañana sin ayer. Batallas por la memoria y consolidación democrática en el Perú. Lima: IEP.

Escolar, C., y Fabri, S. (2009). Memoria y espacio social. La territorialización de la memoria en la construcción de ciudadanía. Revista Panorama 7 (13), 97-109.

Espinoza, V., Ortiz, M., y Rojas, P. (2003). Comisiones de Verdad ¿Un camino incierto? Estudio comparativo de Comisiones de la Verdad en Argentina, Chile, El Salvador, Guatemala y Sudáfrica desde las víctimas y las organizaciones de derechos humanos. Chile: CODEPU - APT.

González, E., y Velásquez, F. (2003). ¿Qué ha pasado con la participación ciudadana en Colombia? Bogotá: Fundación Corona.

Huber, L., y Del Pino, P. (2015). Políticas en Justicia Transicional. Miradas comparativas sobre el legado de la CVR. Lima: IEP. 
IDEHPUCP y Embajada de Francia. (2008). Las reparaciones a las víctimas de la violencia en Colombia y Perú: retos y perspectivas. Lima: BNP.

Janssens, G. (2010). Los lugares de memoria archivística europea: un reconocimiento del recorrido. Pliegos de Yuste 1(11-12), 83-90.

Jelin, E. (2002). Los trabajos de la memoria. Madrid: Siglo XXI.

Jelin, E. (2005). Las luchas por la memoria. Telar. Revista del Instituto Interdisciplinario de Estudios Latinoamericanos (2-3), 17-40.

Levi, P. (2000). Los hundidos y los salvados. Barcelona: Muchnik Editores S.A.

Mora, Y. (2013). Places of memory: between tension, participation and reflection. Revista Panorama 7 (13), 97-109.

Nora, P. (2008). Les Lieux de mémoir. París: Gallimard Editorial.

Reátegui, F. (2010). Los sitios de memoria: procesos sociales de la conmemoración en el Perú. Lima: IDEHPUCP y Fundación Konrad Adenauer.

Rodríguez, J. (2013). Las comisiones de la verdad en el derecho comparado. En M. Requena, Acta V Jornadas de Estudios de Seguridad. Instituto Universitario General Gutiérrez Mellado-UNED (259-290). Madrid: Instituto Universitario General Gutiérrez Mellado.

Rubio, S. (2013). La reparación a las víctimas del conflicto armado en Perú: La voz de las víctimas. Lima: IDL.

Teitel, R. (2003). Genealogía de la Justicia Transicional. Harvard Human Rights Journal (16), 69-94.

Todorov, T. (2000). Los abusos de la memoria. Barcelona: Paidós.

Todorov, T. (2002). Memorial del mal, tentación del bien. Indagación sobre el siglo XX. Barcelona: Península. 